\title{
Implementación sistema de invernadero automatizado para producción de multicultivos agrícolas educativos en el SENA de Mosquera
}

\author{
Adolfo Sebastián Fonseca Luna* y Johan Leonardo Ramos Ramírez ${ }^{* *}$
}

\section{Resumen}

Los invernaderos son instalaciones claves en la producción agrícola. Estos sistemas permiten ejercer un control eficiente de las condiciones atmosféricas, incidencia de plagas y enfermedades, lo que garantiza a los agricultores altos rendimientos, asociados al manejo tecnológico que este sistema permite establecer. Los aprendices de SENA, en su formación educativa integral, deben estar preparados para el manejo, ejecución y capacitación de los procesos entorno al sistema y desarrollo tecnológico de sistema. Por ello, el objetivo de este proyecto es realizar las adecuaciones tecnológicas que permitan el control de condiciones como temperatura, humedad relativa, apertura y cierre de cortinas, sistema de fertirriego, ventiladores y adecuaciones de luz en pro del desarrollo eficiente y sostenible de cultivos agrícolas de gran importancia, para el crecimiento económico del país, el cual está en manos de muchos egresados y estudiantes del SENA.

\begin{abstract}
Greenhouses are key facilities in agricultural production. These controlled systems allow efficient control of atmospheric conditions, incidence of pests, and diseases, which guarantees farmers high yields, associated with the technological management that this system brings. SENA apprentices in their comprehensive educational training must be prepared for the management, execution and training of the processes around the system and technological development of the system. This is why the objective of this project is to carry out the technological adaptations that allow the control of conditions such as: temperature, relative humidity, opening and closing of curtains, fertigation system, fans and light adjustments, in favor of the efficient and sustainable development of agricultural crops. The latter have great importance for the economic growth of the country, which is in the hands of many SENA graduates and students.
\end{abstract}

Cómo citar este artículo (APA): Fonseca y Ramos (2019). Implementación sistema de invernadero automatizado para producción de multicultivos agrícolas educativos en el SENA de Mosquera. Hashtag, 14, 31-38.

> Palabras clave: aprendizaje, automatización, capacitación, desarrollo, economía, invernadero, producción agrícola, tecnología.

$>$ Keywords: agricultural production, automation, development, economy, greenhouse, learning, technology, training.

* Estudiante de Ingeniería Mecatrónica. Contacto: afonsecal@sena.edu.co

** Estudiantes de Ingeniería Mecatrónica. Contacto: johanrr53@gmail.com 


\section{Introducción}

E n Colombia la optimización de los invernaderos desde la fase inicial de diseño ha sido escasa y actualmente no existe ninguna metodología de diseño de estructuras de invernadero, por lo que aún se continúa utilizando la estructura tradicional establecida hace más de 50 años (Bojacá et al., 2011)

(Newman, 1999) reportó que los índices de ventilación de los invernaderos en la sabana de Bogotá son bajos. En la actualidad, aunque se han tomado medidas y generado soluciones, las estructuras de invernadero siguen siendo deficientes en este aspecto. Por esta razón, el proceso de replicar modelos de invernadero genera como consecuencia la construcción de estructuras de invernadero que no tienen la capacidad de proveer las condiciones ambientales óptimas para el desarrollo y crecimiento de los cultivos. En ocasiones, esta situación puede crear condiciones ambientales adversas generadas por una ineficiente ventilación que se traduce en elevadas temperaturas diurnas, deficiencias de $\mathrm{CO} 2$, humedades variables y una pérdida acelerada de la energía almacenada durante el día, en las horas de la noche.

El diferencial térmico entre el día y la noche generado bajo esta condición favorece la aparición y desarrollo de problemas fitosanitarios (Cooman y Lee, 1998). En los últimos años, los invernaderos para la producción agrícola se han

\section{Metodología}

Con el fin de elaborar el diseño del prototipo de un invernadero con un sistema automatizado, se consideran tres factores principales. Estos son:

1. Mantener las condiciones de temperatura y humedad. convertido en una herramienta importante para los agricultores, al permitirles alcanzar objetivos de sostenibilidad, productividad y optimización de los recursos.

La automatización de los invernaderos permite reducir costos de producción, optimizar los recursos, principalmente el agua, mediante la implementación de sistemas de riegos, reducir las aplicaciones químicas por presencia de plagas y/o enfermedades. Estos proyectos se diseñan con el fin de ahorrar considerablemente el agua que se utiliza en el riego de plantas (Berenz, 2007).

Los aprendices del Centro de Biotecnología Agropecuaria del Servicio Nacional de Aprendizaje, SENA, en particular los estudiantes de programas técnicos y tecnológicos en producción agrícola y afines, cuentan con un invernadero tipo cubierta que no se encuentra automatizado, por lo que los ensayos y prácticas institucionales no se enfocan en la importancia de este componente tecnológico, para los estudiantes en formación y conocimiento del sector agrícola.

El objetivo del proyecto, del cual se deriva este artículo, es realizar la automatización de una instalación tipo cubierta que se encuentra en el SENA, enfocado a la realización del sistema de riego, instalación de sensores, ventiladores y software de almacenamiento y control de la información.

2. Establecer un protocolo de registros, bitácoras de seguimiento continuo, además de la vigilancia y supervisión del sistema.

3. Controlar con eficacia el sistema de riego y de ventilación 
4. La manipulación de la interfaz es de fácil entendimiento para que resulte útil el sistema automatizado para cualquier individuo.

5. Realizar el mantenimiento trimestral de los equipos y diferentes materiales que componen el sistema.

\section{Materiales}

- Ventiladores

- Cintas de riego

- Conectores manguera-cinta

- Conectores manguera-manguera
- Dispersores

- Válvulas de control

- Programador digital

- Estación de bombeo

- Sensores (Temperatura \& Humedad Relativa)

- Tensiómetro (Medidor de agua en el suelo)

- Inyector fe fertilizantes por lámina de riego

- Software receptor de información diaria.

Asimismo, se requiere de personal capacitado para el establecimiento y ajuste del sistema.

\section{Configuración de sensores y controles electrónicos}

Con el fin de mantener todo el sistema en comunicación, se escoge el microcontrolador arduino, que se compone por una plataforma llamada
ATmega328. Esta contiene contiene un sistema electrónico abierto basado en un hardware y software libre, flexible y fácil.

Figura 1. Microcontrolador arduino uno-r3.

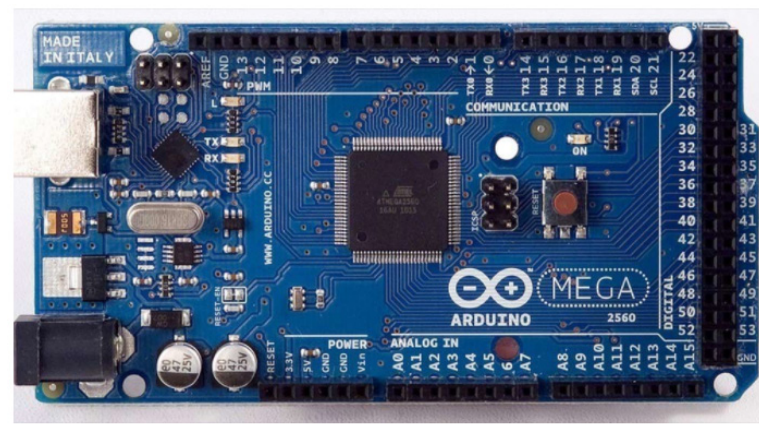

Fuente: Arduino Uno-R3

Este microcontrolador cuenta con las siguientes características:

- Microcontrolador ATmega 328

- Voltaje de entrada 7-12V

- 14 pines digitales de I/O con 6 salidas PWM
- 6 entradas análogas

- 32k de memoria Flash

- Reloj de $16 \mathrm{MHz}$ de velocidad

El uso de esta tarjeta permite acceder al adecuado manejo de los sistemas de riego, sistemas de ventilación, humedad relativa y calidad del 
aire con el fin de mantener las condiciones ideales de los cultivos, en este caso de hortalizas, con el fin de mejorar las respuestas en cuanto a rendimiento, precocidad, calidad y cantidad del producto a explotar. Partiendo de la información adquirida los sensores recomendados para el control y monitoreo frente al manejo de las condiciones del invernadero recomendadas son:

\section{Sensor DHT1 1}

El sensor digital DHT11 monitorea la temperatura y la humedad relativa. Este producto "integra un sensor capacitivo de humedad y un termistor para medir el aire circundante, y muestra los datos mediante una señal digital en el pin de datos (no posee salida analógica). Utilizado en aplicaciones académicas relacionadas al control automático de temperatura, aire acondicionado, monitoreo ambiental en agricultura y más" (Suarez, 2019). Este sensor integra los pines analógicos del Arduino y los analiza y los procesarla dando orden a los diferentes actuadores como los ventiladores y extractores obteniendo una mejor productividad. Este producto cuenta con las siguientes características:

- Rango de voltaje de 3V a 5V

- Rangos: medición de temperatura $0^{\circ} \mathrm{C}-50^{\circ} \mathrm{C}$ y humedad relativa $20 \%-90 \%$

- Presenta compatibilidad con tarjetas Arduino

- Pin de salida digital con dimensiones de tarjeta de $3.2 \mathrm{~cm} \times 1.4 \mathrm{~cm}$

Figura 2. Sensor DHT11.

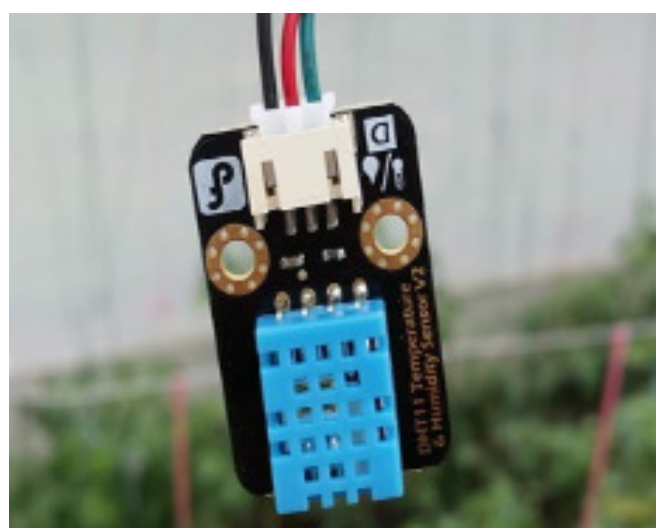

Fuente: Rincón, Torres Silva. (2016).

\section{Sensor Watermark 200TS}

Es un sensor que tiene como función medir la humedad del suelo, con el fin de hacer un control en cuanto a la frecuencia y la dosis para la adecuada administración del riego teniendo en cuenta los requerimientos hídricos por cultivo. Esto se logra a través de las señales digitales y análogas que envía este sensor, obtenidas directamente del invernadero. Dichas señales son procesadas por Arduino, lo que ocasiona el funcionamiento de las válvulas y el riego controlado. Para asegurar el adecuado funcionamiento de este sensor se requiere un adaptador Watermark 220SS-VA, que evalúa las presiones del suelo húmedo, para la captación de los datos en cuanto a humedad y temperatura, por medio de salidas con datos análogos. 
Figura 3. (A) Sensor de temperatura, (B) Sensor watermark, (C) Adaptador watermark.
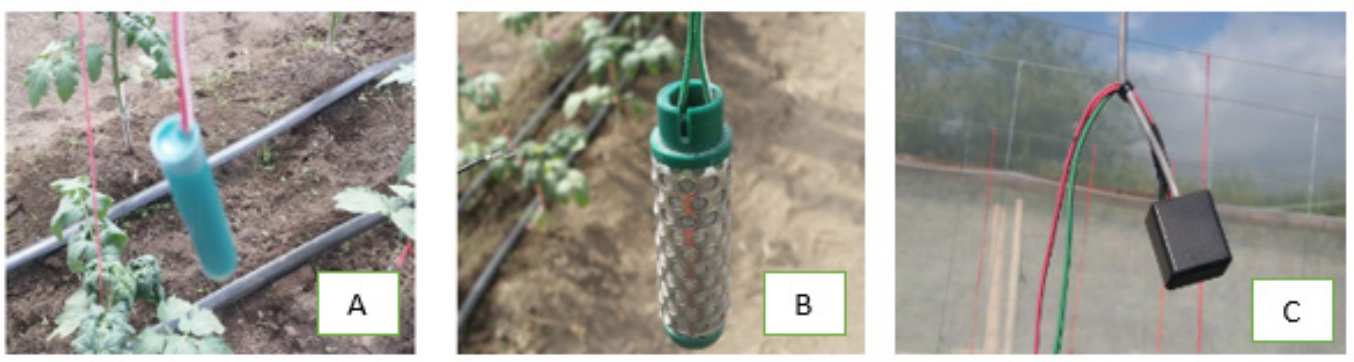

Fuente: Rincón, Torres, Silva. (2016).

Por otro lado, se cuenta con un sensor que mide la humedad presente en el suelo. Este es el sensor FC-28, encargado de medir la conductividad. De igual forma, va conectado al adaptador
Waltermark, con el fin de recibir señales con datos análogos para detectar cuándo es necesario activar el sistema de bombeo.

Figura 4. Sensor FC-28.

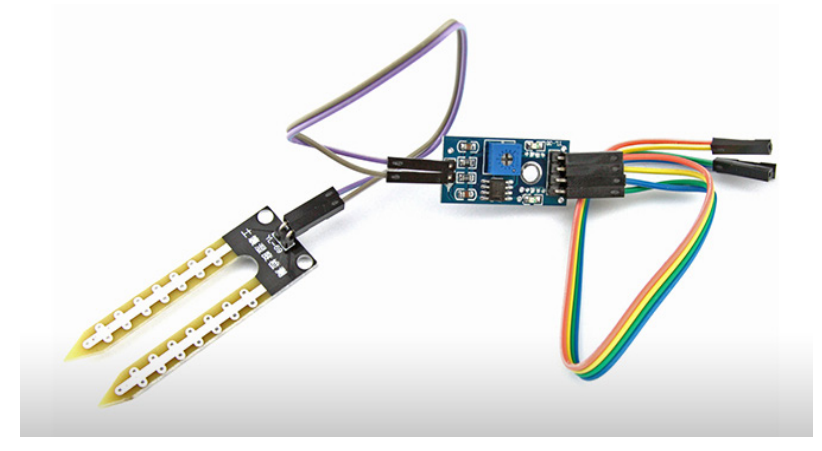

Fuente: Rincón, Torres y Silva. (2016).

\section{Sistema de riego}

El sistema de riego que fue implementado en el invernadero fue por goteo, debido a que su método de aplicación de agua es controlado directamente en la zona radicular. Además, resulta de fácil uso en el momento de aplicar diversos fertilizantes, lo que incrementa el aprovechamiento de estos, debido a su aplicación en la zona radicular de las hortalizas.

Para el montaje en campo se tiene en cuenta la necesidad de emplear dos cintas de riego por surco. Estas cintas cuentan con una capacidad de 2 1/hora, con disposición de goteros cada $15 \mathrm{~cm}$. Este sistema de riego cuenta con una electrobomba con una capacidad de $3 / 4 \mathrm{Hp}$ y un tanque de $1000 \mathrm{~cm}^{3}$. Para la inyección adecuada de los fertilizantes, se cuenta con una malla de $1 \mathrm{ft}$ para evitar la obstrucción de las cintas de riego. Su sistema de control se compone por 2 muñecas en PVC con válvula plástica. 
Figura 5. Sistema de riego por goteo con doble cinta por surco.

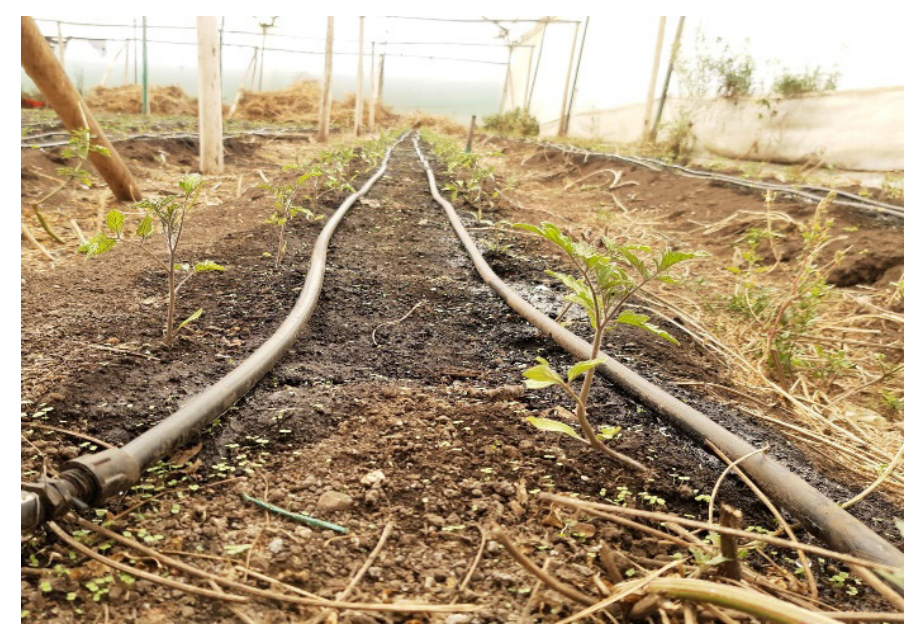

Fuente: elaboración propia.

Con el fin de elaborar este sistema automatizado, se necesita un control del sistema de riego por medio de relés y electroválvulas diseñadas con el fin de enviar mensajes al microcontrolador Arduino, por medio del primer relé. Esto ocurre cuando los niveles de humedad presentes en el suelo son bajos. Este trabajo en conjunto se da por la primera actividad hecha por el relé, ya que a través de un campo magnético se acciona un interruptor que da directamente con una bobina. Esta envía señales a las electroválvulas para abrir o cerrar en caso de que sea necesario, partiendo por los datos obtenidos respecto a la humedad presente en el suelo.

Figura 5. (A) Eletroválvula, (B) Relé de 5v.
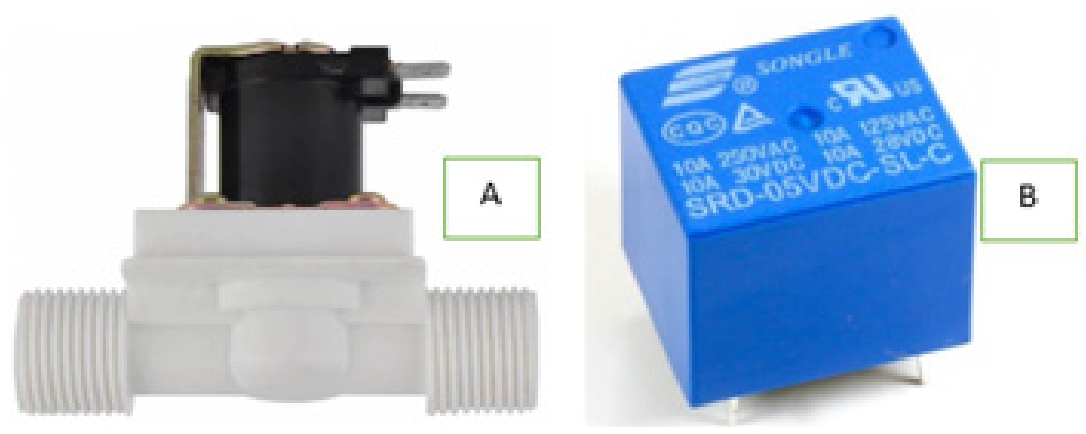

Fuente: Electronilab.

\section{Sistema de ventilación}

Con el fin de garantizar una producción estable bajo condiciones de invernadero, es necesario contar con sistemas de extractores para el manejo de la temperatura y humedad, para evitar daños en el cultivo, pues estos representan una baja en la calidad y cantidad de la producción. 
Para asegurar el mantenimiento adecuado, se decide hacer uso automatizado de extractores. Esta automatización se logra por medio del uso de relés, extractores y contactores que envían señales a los microcontroladores arduinos, con el fin de encender estos extractores cuando los datos análogos lo pidan. De esta forma se asegura el mantenimiento de la temperatura adecuada para los diferentes cultivos de hortalizas situadas en este invernadero. Esta acción se trabaja de la mano con los sensores DHT11, que también se encargan de enviar señales análogas, como se explicó anteriormente (figura 2).

Figura 6. Ventilador activo

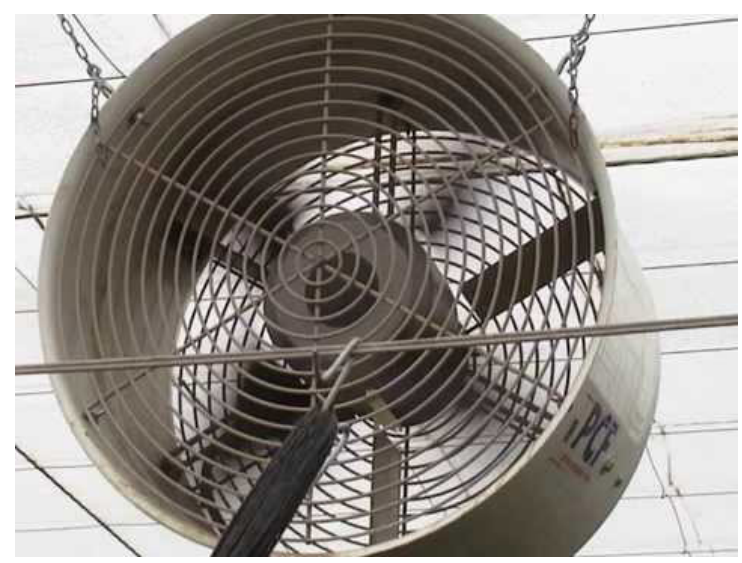

Fuente: elaboración propia.

\section{Resultados}

La implementación de sistemas automatizados en el invernadero con fines educativos en la sede del SENA en Mosquera demuestra ser altamente viable, tanto en el aspecto educativo como en el productivo. En lo referente al ámbito educativo, se demuestra interés por parte de los desde el momento del ensamblaje de todo el sistema, hasta su manipulación. A nivel productivo se obtienen buenos resultados, debido a que, con un adecuado ensamblaje de cada componente perteneciente a este sistema, se logra obtener datos análogos que son mostrados, controlados y almacenados por medio de sistemas operativos en dispositivos móviles o computadores. Esto también permite tener el control en determinado momento, cuando este sistema nos muestre alguna posible falla en conexiones o en el cultivo, de manera que se tomen las medidas necesarias y a tiempo, lo que deriva en la disminución de posibles pérdidas en la producción.

Este control y almacenamiento de datos se logró por medio de los microcontroladores aportados por el sistema de Arduino, cuya comunicación se logra por medio de bluetooth y permite enviar los datos "en vivo" de la humedad relativa, temperatura y humedad del suelo presentes en el invernadero. También permite guardar el histórico de estos datos con el fin de hacer posibles proyecciones y ajustes cuando sea necesario. Por otro lado, la sistematización de las aplicaciones de riego o fertirriego benefician la calidad de aplicación y optimización del tiempo de parte de los estudiantes, pues pueden desarrollar otras actividades dentro del invernadero mientras el riego o fertirriego es aplicado por el sistema. 


\section{Conclusiones}

A manera de conclusión, se puede decir que la automatización de los invernaderos demuestra tener viabilidad en cuanto a que son herramientas que benefician a los productores, ya que se les permite tener información detallada que puede ser controlada por medio de dispositivos móviles.

Además, la automatización se puede realizar en cualquier invernadero con el fin de implementar y tecnificar los diversos cultivos en los que se pueden usar, ya que su automatización presenta bajos costos y puede resultar en altos rendimientos, productividad y calidad de los productos a desarrollar.

Por último, los aprendices de carreras técnicas y tecnológicas enfocadas a la producción agrícola del SENA de Mosquera tendrán la posibilidad de acceder a una formación integral. Los invernaderos automatizados complementan los aspectos teóricos y el conocimiento del manejo del sistema fortalecerá sus capacidades para próximas etapas laborales.

\section{Referencias}

Berenz, G (2007). Lectura remota de las variables de un invernadero usando telemetría. Recuperado de http://www.radiocomunicaciones.net/pdf/telemetria/lecturaremota-invernadero-telemetria.pdf

Bojacá C.R., Gil, R., Schrevens, E. (2011). The greenhouse effect in the high tropics of Colombia: a modeling approach. Acta Horticulturae 893, 791- 796.

Cooman. A. Y., Lee. R. Investigacion en control de clima en la Sabana de Bogota, las condiciones locales y sus limitaciones. Plantflor Cultivo E Comercio, 2:41-43, 1998. [50]. Recuperado de https:/ / repositorio.unal.edu.co/bitstream/handle/unal/56572/1072644298.2016. pdf? sequence $=1 \&$ isAllowed $=y$

Newman (1999). Efectos del clima en la fisiología de la floración. En clima fisiología y producción de cultivos bajo invernadero, LEE, Rebecca. Santafé de Bogotá centro de investigaciones y asesorías agroindustriales. 1999. p 15-29 Recuperado de https:/ / repositorio.unal.edu.co/ bitstream/handle/unal/56572/1072644298.2016.pdf?sequence=1\&isAllowed=y

Suarez G. (23 de febrero de 2019). Naylamp Mechatronics. (Naylamp Mechatronics SAC) Recuperado de https://naylampmechatronics.com/sensores-temperatura-y-humedad/57-sensor-de-temperatura-y-humedad-relativa-dht11.html 